\title{
Transient Attosecond Soft-X-Ray Spectroscopy in Layered Semi-Metals
}

\author{
T. P.H. Sidiropolous ${ }^{1}$, N. Di Palo ${ }^{1}$, D. E. Rivas ${ }^{1,2}$, S. Severino ${ }^{1}$, M. Reduzzi ${ }^{1}$, B. Buades ${ }^{1}$, I. Leon ${ }^{1}$, S. L. \\ Cousin $^{1}$, M. Hemmer ${ }^{1}$, C. Cocchi ${ }^{3}$, E. Pellegrin ${ }^{4}$, J. Herrero Martin ${ }^{4}$, S. Mañas-Valero ${ }^{5}$, E. Coronado ${ }^{5}$, \\ T. Danz ${ }^{6}$, C. Draxl ${ }^{3}$, M. Uemoto ${ }^{7}$, K. Yabana ${ }^{7}$, M. Schultze ${ }^{8}$, S. Wall ${ }^{1}$, A. Picon ${ }^{1,9}$, J. Biegert ${ }^{1,10}$ \\ 1. ICFO-Institut de Ciencies Fotoniques, E-08860 Castelldefels, Barcelona, Spain \\ 2. European XFEL GmbH, Holzkoppel 4, D-22869 Schenefeld, Germany \\ 3. Institut für Physik and IRIS Adlershof, Humboldt-Universität zu Berlin, Berlin, Germany \\ 4. ALBA Synchrotron Light Source, E-08290 Cerdanyola del Vallès, Barcelona, Spain \\ 5. Instituto de Ciencia Molecular (ICMol), Universitat de València, Paterna, Spain \\ 6. 4th Physical Institute - Solids and Nanostructures, University of Göttingen, Göttingen, Germany \\ 7. Center for Computational Sciences, University of Tsukuba, Tsukuba 305-8577, Japan \\ 8. Fakultät für Physik, Ludwig-Maximilians- Universität, Am Coulombwall 1, D-85748 Garching, Germany \\ 9. Departamento de Química, Universidad Autónoma de Madrid, 28049 Madrid, Spain \\ 10. ICREA, Pg. Lluís Companys 23, 08010 Barcelona, Spain
}

X-ray absorption fine-structure (XAFS) spectroscopy is a well-established technique capable of extracting information about a material's electronic and lattice structure with atomic resolution. While the near-edge region (XANES) of a XAFS spectrum provides information about the electronic configuration, structural information is extracted from the extended XAFS (EXAFS) spectrum, consisting of several hundreds of eV above the absorption edge. With the advent of high harmonic sources, reaching photon energies in soft X-ray (SXR) region, it now becomes possible to connect the spectroscopic capabilities of XAFS to the unprecedented attosecond temporal resolution of a high harmonic source allowing the observation of electronic and lattice dynamics in real time [1,2].

Layered materials, such the transition-metal dichalcogenide $\mathrm{TiS}_{2}$ or graphite, are an emerging class of materials with attractive structural and electronic properties as they can be thinned to a single atomic layer with electron mobilities resembling that of a metal, semiconductor, or semi-metal.

In this work, we utilized broadband water-window-covering attosecond SXR pulses ( 300 as, ranging from 200 - $550 \mathrm{eV}$ ) capable of accessing orbital-specific K- and L-edges of such layered materials to perform transient XAFS with attosecond time resolution [3,4].

[1] Teichmann, S. et al, "0.5-keV soft x-ray attosecond continua", Nat. Commun. 7, 11493 (2016).

[2] Cousin S. et al, "Attosecond streaking in the water window: a new regime of attosecond pulse characterization", Phys. Rev. X, 7, 041030 (2017).

[3] Buades, B. et al., "Dispersive soft X-ray absorption fine-structure spectroscopy in graphite with an attosecond pulse", Optica 5 (5), 502 (2018).

[4] Buades, B. et al., “Attosecond-resolved petahertz carrier motion in semi-metallic TiS2”, arXiv: 1808.06493 (2018). 